\title{
SMS text pre-notification and delivery of reminder e-mails to increase response rates to postal questionnaires: a factorial design, randomised, controlled trial
}

\author{
Kath Starr, Gladys McPherson, Mark Forrest, Seonaidh Cotton* \\ From 3rd International Clinical Trials Methodology Conference \\ Glasgow, UK. 16-17 November 2015
}

\section{Background}

Participant non-response to postal questionnaires in randomised controlled trials (RCT) can jeopardise trial results.

\section{Methods \\ We evaluated two interventions aimed at increasing postal questionnaire response rates in a $2 \times 2$ partial factorial RCT nested within a single, large, UK-wide RCT of medical expulsive therapy for ureteric stones. The interventions were: questionnaire pre-notification via SMS text prior to posting the trial questionnaires versus no pre-notification; for non-responders to the initial questionnaire mailing, use of an e-mail reminder (containing a hyperlink for online questionnaire completion) versus a postal reminder with paper questionnaire. Participants were randomised to the pre-notification comparison, the reminder comparison or both, depending on whether they supplied mobile tele- phone number and/or email address. The primary out- come was response to 4 and 12 week questionnaires.}

\section{Results}

418 participants were randomised to the SMS pre-notification comparison: the intervention had no effect on response rates at either questionnaire time point. In sub-group analyses, SMS pre-notification increased response rates in women $(\mathrm{p}=0.038)$, but only at the first time-point. 119 participants were randomised to the reminder comparison. There was no difference in response rates between those randomised to e-mail or postal reminders.

University of Aberdeen, Aberdeen, UK 